\title{
Erica Durante, Poétique et écriture: Dante au miroir de Valéry et de Borges
}

\section{Andrea Schellino}

\section{(2) OpenEdition}

1 Journals

\section{Edizione digitale}

URL: http://journals.openedition.org/studifrancesi/5816

DOI: ERREUR PDO dans /localdata/www-bin/Core/Core/Db/Db.class.php L.34 : SQLSTATE[HY000]

[2006] MySQL server has gone away

ISSN: 2421-5856

\section{Editore}

Rosenberg \& Sellier

\section{Edizione cartacea}

Data di pubblicazione: 1 septembre 2011

Paginazione: $477-478$

ISSN: 0039-2944

\section{Notizia bibliografica digitale}

Andrea Schellino, «Erica Durante, Poétique et écriture: Dante au miroir de Valéry et de Borges», Studi Francesi [Online], 164 (LV | II) | 2011, online dal 30 novembre 2015, consultato il 08 janvier 2021. URL: http://journals.openedition.org/studifrancesi/5816 ; DOI: https://doi.org/ERREUR PDO dans / localdata/www-bin/Core/Core/Db/Db.class.php L.34 : SQLSTATE[HY000] [2006] MySQL server has gone away

Questo documento è stato generato automaticamente il 8 janvier 2021.

\section{(c)}

Studi Francesi è distribuita con Licenza Creative Commons Attribuzione - Non commerciale - Non opere derivate 4.0 Internazionale. 


\title{
Erica Durante, Poétique et écriture: Dante au miroir de Valéry et de Borges
}

\author{
Andrea Schellino
}

\section{NOTIZIA}

ERICA DURANTE, Poétique et écriture: Dante au miroir de Valéry et de Borges, Paris, Honoré Champion Éditeur, 2008, 549 pp.

«L'enorme costruzione attuata da secoli di critica su un classico, nel nostro caso Dante, diverrebbe intollerabile per uno spirito sano, se egli non potesse rifugiarsi ogni tanto in quegli ariosi edifici che sono le letture poetiche che di un poeta fa un altro poeta» (cit., p. 17). Da quest'affermazione di Maria Corti prende spunto il libro di Erica DURANTE, sviluppo di una tesi di dottorato sostenuta nel 2004 all'Université Sorbonne NouvelleParis III, che affronta con scrupolo e sensibilità le letture (e soprattutto le scritture che ne sono conseguite) che Paul Valéry e Jorge Luis Borges hanno dato di Dante, attraverso una prospettiva teorica sapientemente bilanciata tra genetica testuale e riflessione comparatista. Questa analisi diviene così occasione per mostrare legami inattesi tra i due scrittori, entrambi costantemente appellati dalla voce del poeta fiorentino, nonché per interrogarsi sulla rilevanza della poesia dantesca nell'intera modernità. Per l'A. proprio in virtù di questo dialogo vivace e persistente con Dante, Valéry e Borges trovarono uno stimolo essenziale per affrontare il nucleo e il senso stesso della scrittura e del ruolo del poeta: «L'un, doué d'une "lucidité organisatrice", l'autre, doué d'une "lucidité associative", Valéry et Borges ont l'occasion de confronter leurs deux rapports différents à la littérature grâce à la Comédie» (p. 391). Sotto un certo punto di vista, prima dell'apparizione, nel 1965, del numero dedicato a Dante della rivista Tel Quel, Valéry e Borges riconobbero che, come scrisse Philippe Sollers, il vero mistero di Dante è il rapporto profondo che questi intrattiene con la scrittura e con il linguaggio, al punto che è possibile aggiungere ai quattro sensi indicati dal poeta nella lettura della Commedia, un quinto, il «sens hyper-littéral». Tra le scelte metodologiche cruciali dell'A., presentate nell'Introduction générale, spicca quella di accordare particolare attenzione 
alle attitudini e alle reazioni di lettura di due scrittori, privilegiando esplicitamente la poesia come luogo d'incontro intimo e prezioso; una scelta senza dubbio interessante per ciò che concerne Borges, del quale è stato spesso studiato al contrario il rapporto con Dante sotto altri aspetti.

2 Il libro è strutturato in tre parti; le prime due (rispettivamente La voix de Dante dans l'écriture de Paul Valéry e La voix de Dante dans la poésie de Jorge Luis Borges) analizzano indipendentemente e in modo diacronico le traiettorie dantesche di Valéry e Borges, nonché la presenza del Fiorentino nei due scrittori. La terza parte (Épilogue), invece, è uno studio comparato degli itinerari danteschi di Valéry e Borges, dal quale emergono i cammini poetici ed esegetici, a volte divergenti, a volte contigui o coincidenti, dei due scrittori: «Dante, qui agit pour Valéry et pour Borges comme un déclencheur de possibilités et de convocations multiples à l'intérieur de la littérature, fait apparaître des liens inattendus entre les deux écrivains, là où, de prime abord, on ne voit que des différences» (p. 391).

In un secolo e in una nazione spesso refrattari a Dante, per l'A. Paul Valéry è stato tra coloro, al di là delle sue origini italiane che lo predisponevano a questo contatto, che più si sono sentiti toccati dal poeta fiorentino, tanto da procedere a una lenta ma radicale assimilazione nella sua scrittura. Già nei suoi debutti poetici, Valéry integra talvolta in esergo ai suoi componimenti citazioni dantesche, come avviene in Église de couvent e in Le vent, roi de l'Espace, immole les grands chênes; risonanze ed echi della Commedia si ritrovano anche in En route e Fleur mystique. Una sperimentazione formale dettata dalla lectio dantesca s'impone in La Fileuse, in cui Valéry ricorre per l'unica volta nella sua scrittura alla terza rima o rima incatenata, seguendo l'esempio del poema dantesco; tuttavia, il modello ritmico e formale della Commedia influenza più di ogni altro componimento valéryano il Cimetière marin: il decasillabo utilizzato dal poeta francese discende chiaramente dall'endecasillabo dantesco.

Dopo il ritorno alla poesia dello scrittore francese, secondo l'A. Dante assume per Valéry un ruolo di primissimo piano; è infatti l'esempio mirabile di un felice equilibrio poetico ottenuto grazie alla sovranità del suo spirito, che lo fa essere un grande poeta della conoscenza. In questo modo Valéry pensa di aver raggiunto con il Cimetière marin la «Comédie de l'Intellect» che aveva auspicato affiancarsi a quella umana di Balzac e divina di Dante. Insomma, in Valéry si assiste a un fenomeno notato da altri due eccellenti lettori della Commedia, Percy Bysshe Shelley e Thomas Stearns Eliot, per i quali l'influenza di Dante, laddove è potente, è un'influenza cumulativa, destinata a crescere negli anni e con la maturità della propria scrittura. Accanto a questa assimilazione poetica, che durerà fino agli ultimi anni di vita di Valéry, si situa per Erica Durante la forte impronta reale che assume l'opera dantesca nella vita del poeta francese: da un lato la Vita Nuova dell'idillio di Montpellier, dall'altro la liaison con Catherine Pozzi, sua autentica Beatrice.

5 Rispetto a quella valéryana, la lectura dantis borgesiana è per l'A. più costante e fondamentalmente scevra dalle riserve che Valéry talvolta espresse sul poeta fiorentino; Borges non tarderà a giudicare Dante l'emblema della perfezione e della pienezza poetica e, verso la fine della sua vita, dichiarerà che «todos derivamos de Dante, no es posible literatura sin Dante». Questo apprezzamento, formulato secondo un'ottica poetica, informa nel filo degli anni le numerose composizioni borgesiane in cui è facile cogliere i marchi danteschi; tra queste spiccano le riscritture della Commedia composte da Borges tra il 1942 e il 1979: Del infierno y del cielo, Poema conjectural, Paradiso 
XXXI, 108, Inferno, I, 32 e Inferno, V, 129. A precedere questa densa attività poetica era stata in Borges un'altrettanto intensa esplorazione bibliografica e soprattutto una ricca stagione d'analisi critica, i cui luoghi prediletti sono per l'A. la polivocità della Commedia (rivendicata dal poeta fiorentino medesimo nell' Epistola XII o Epistola a Cangrande), il sospetto - refrain di diversi saggi borgesiani su Dante - e l'humana pietas che il poeta manifesta a volte verso i dannati.

6 Per l'A., infine, è importante notare che lo sbocco ultimo della riflessione dantesca di Borges consiste nella formulazione del principio di una matrice unica e universale della letteratura: la Commedia non è solo il capriccio isolato di un individuo, ma piuttosto lo sforzo di molti uomini e generazioni; in essa convergono movimenti, avventure, intuizioni e premonizioni dello spirito umano.

7 Il volume presenta al lettore una ricca documentazione secondaria, che contiene tra l'altro un facsimile di alcune annotazioni di lettura di Valéry della Commedia, le referenze dantesche nell'opera del poeta francese e testi provenienti dal fondo Catherine Pozzi della Bibliothèque nationale de France. 